\title{
ERREUR DUE AU TARIF DANS LE CALCUL DU VOLUME DES ÉCLAIRCIES A PARTIR DE LA DIMENSION DES SOUCHES
}

\author{
N. DECOURT * \\ avec la collaboration de M. AyraL ** \\ Station de Sylviculture et Production \\ Centre national de Recherches forestières, I.N.R.A., \\ Champenoux 54370 Einville
}

\section{RÉSUMÉ}

Le volume inconnu d'une éclaircie passée dont on ne retrouve que les souches, ne peut être estimé valablement (biais faible, erreur inférieure à $10 \%$ ) que si elle a eu lieu il y a moins de 5 ans pour les peuplements jeunes. Au-delà, cette estimation n'est possible que dans les peuplements âgés, à croissance faible.

\section{I. - POSITION DU PROBLÈME}

Dans les placettes temporaires destinées à l'évaluation de la production, il est intéressant de connaître non seulement le volume sur pied, mais également le volume de bois enlevé en éclaircie avant la date de la mesure. Cette opération est nécessaire pour essayer d'approcher, même par défaut, la production totale et donc l'accroissement moyen annuel qu'on sous-estimerait beaucoup en ne considérant que le peuplement sur pied. De plus, évaluer le volume d'une coupe, à partir des souches restantes peut avoir un intérêt pour diverses expertises, dans les cas de délits par exemple.

La méthode employée consiste à faire l'inventaire des souches, en diamètre ou en circonférence, et à s'appuyer sur la relation linéaire bien connue (PARdé 1961, DeCOURT 1964) qui relie la circonférence à hauteur de souche à la circonférence à $1,30 \mathrm{~m}$. On utilise soit une relation moyenne, soit plus souvent une relation calculée à partir d'un échantillon de tiges sur pied sur lesquelles on mesure ces deux circonférences. La méthode n'est appliquée que pour les peuplements purs et équiennes. Il n'est pas exclu, ce qui serait à vérifier, qu'elle soit valable dans les peuplements inéquiennes.

Cette relation permet de restituer à partir du comptage et de la mesure des souches l'inventaire en circonférence à $1,30 \mathrm{~m}$ des arbres enlevés par les éclaircies.

Pour passer au volume, on utilise alors un tarif valable pour le peuplement sur pied au moment de la mesure. Pour les peuplements purs et équiennes, on établit souvent ce tarif, à partir d'un échantillon de tiges du peuplement, en ajustant une équation de la forme;

$$
v=a+\mathrm{bC}^{2}
$$

reliant linéairement le volume d'une tige $(v)$, au carré de sa circonférence à $1,30 \mathrm{~m}(\mathrm{C}) ; a$ et $b$ étant des constantes spécifiques du peuplement étudié et dépendant entre autre de l'essence, de la station et de l'âge.

Il est bien connu notamment que ces tarifs « montent » avec l'âge, c'est-à-dire que toutes choses égales par ailleurs, pour une même circonférence, le volume est d'autant plus fort que l'âge est plus grand.

(*) Maître de Recherches Station de Sylviculture et de Production, C.N.R.F.

(**) Station de Sylviculture et Production du C.N.R.F., décédé le 27 octobre 1970. 
Les éclaircies, étant intervenues souvent plusieurs années avant la mesure, on utilise donc pour évaluer leur volume par cette méthode un tarif trop « fort » et on a donc tendance, en théorie, à surestimer leur volume.

Les erreurs dans cette récupération des éclaircies sont donc dues essentiellement :

- au difficile comptage des souches et à leurs mensurations souvent imprécises, notamment pour les souches anciennes plus ou moins dégradées.

- à l'imprécision de la relation permettant le passage de la circonférence de la souche à la circonférence à $1,30 \mathrm{~m}$. Nous avons cependant montré dans une précédente publication (DECOURT 1964) que cette relation est en général excellente, et que la source principale d'erreur n'est pas là.

- à l'utilisation d'un tarif inadéquat. Cette source d'erreur, très importante comme nous le verrons, n'a pas été étudiée à notre connaissance, et nous essayons ici d'en estimer l'ordre de grandeur.

\section{II. - MÉTHODE D'ÉTUDE}

Notre étude n'a porté que sur des peuplements résineux, équiennes et purs. Elle est basée sur les données fournies par les placettes permanentes de la station de Sylviculture et Production du C.N.R.F,

Dans ces placettes, les peuplements sur pied sont cubés, à partir d'un échantillon de tiges, par le calcul d'un tarif en $\mathrm{C}^{2}$, comme nous l'avons indiqué ci-dessus. Les éclaircies sont cubées tige par tige, avec grand soin.

On dispose done d'un certain nombre de tarifs établis à un âge donné $\mathrm{A}_{o}$ et du volume réel $\left(\mathrm{V}_{\mathrm{r}}\right)$ des éclaircies effectuées $\Delta \mathrm{A}$ années avant, à des âges variés, tels que $\mathrm{A}=\mathrm{A}_{0}-\Delta \mathrm{A}$.

Il est alors facile d'évaluer le volume de ces éclaircies à l'aide du tarif valable à l'âge $A_{o},\left(V_{t}\right)$. On calculera ensuite l'erreur relative (e) :

$$
e=\frac{\mathrm{V}_{\mathrm{r}}-\mathrm{V}_{\mathrm{t}}}{\mathrm{V}_{\mathrm{r}}}
$$

Pour avoir une idée de la dispersion de ces erreurs, on calculera les écarts-types $(s)$ des $e$ et on cherchera à voir si la moyenne des erreurs est ou non significativement différente de zéro; c'est-à-dire, si comme on s'y attend l'erreur $e$ est biaisée négativement.

Les éclaircies étudiées sont réparties comme l'indique le tableau I, les âges au moment de l'éclaircie s'étendent de 20 à 160 ans *.

TABLEAU 1

Répartition des éclaircies étudiées par espèces et par décalage avec "l'âge 》 du tarif $(\Delta \mathrm{A})$

\begin{tabular}{|c|c|c|c|c|c|c|c|c|}
\hline$\underset{\text { Espèces }}{\Delta \mathrm{A}}$ & $0-4$ & $5-9$ & $10-14$ & $15-19$ & $20-24$ & $25-29$ & $30-34$ & Total \\
\hline Pin maritime..$\ldots \ldots$ & 10 & 6 & 8 & 2 & 4 & 4 & - & 34 \\
\hline Pin noir $\ldots \ldots \ldots \ldots$ & 11 & - & 8 & 2 & 6 & - & 1 & 28 \\
\hline Epicéa ........... & 11 & 2 & 4 & - & 2 & 4 & - & 23 \\
\hline Cèdre $\ldots \ldots \ldots \ldots$ & 4 & - & 2 & - & 2 & - & - & 8 \\
\hline Mélèze du Japon .... & 6 & 5 & 2 & 1 & 1 & 1 & - & 16 \\
\hline Pin sylvestre $\ldots \ldots \ldots$ & 50 & 12 & 16 & 9 & 12 & 15 & 3 & 117 \\
\hline Sapin $\ldots \ldots \ldots \ldots$ & 52 & 13 & 20 & 9 & 12 & 5 & 4 & 115 \\
\hline Douglas $\ldots \ldots \ldots \ldots$ & 4 & 3 & 3 & 3 & 3 & 3 & - & 19 \\
\hline TOTAL & 148 & 41 & 63 & 26 & 42 & 32 & 8 & 360 \\
\hline
\end{tabular}

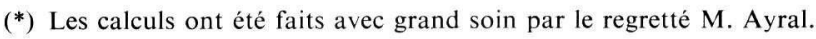




\section{III. - RÉSULTATS}

\section{1. - Influence de l'espèce}

Il ne semble pas que les différentes espèces étudiées se comportent très différemment les unes des autres, ni quant au biais qui croît avec le décalage $\Delta \mathrm{A}$ entre la date d'établissement du tarif et la date de l'éclaircie, ni quant à la dispersion des erreurs à craindre qui est considérable. Ce fait est particulièrement remarquable dans le cas du sapin et du pin sylvestre, essences pour lesquelles on dispose d'un nombre de données suffisant. La figure 1, illustre parfaitement ce phénomène.

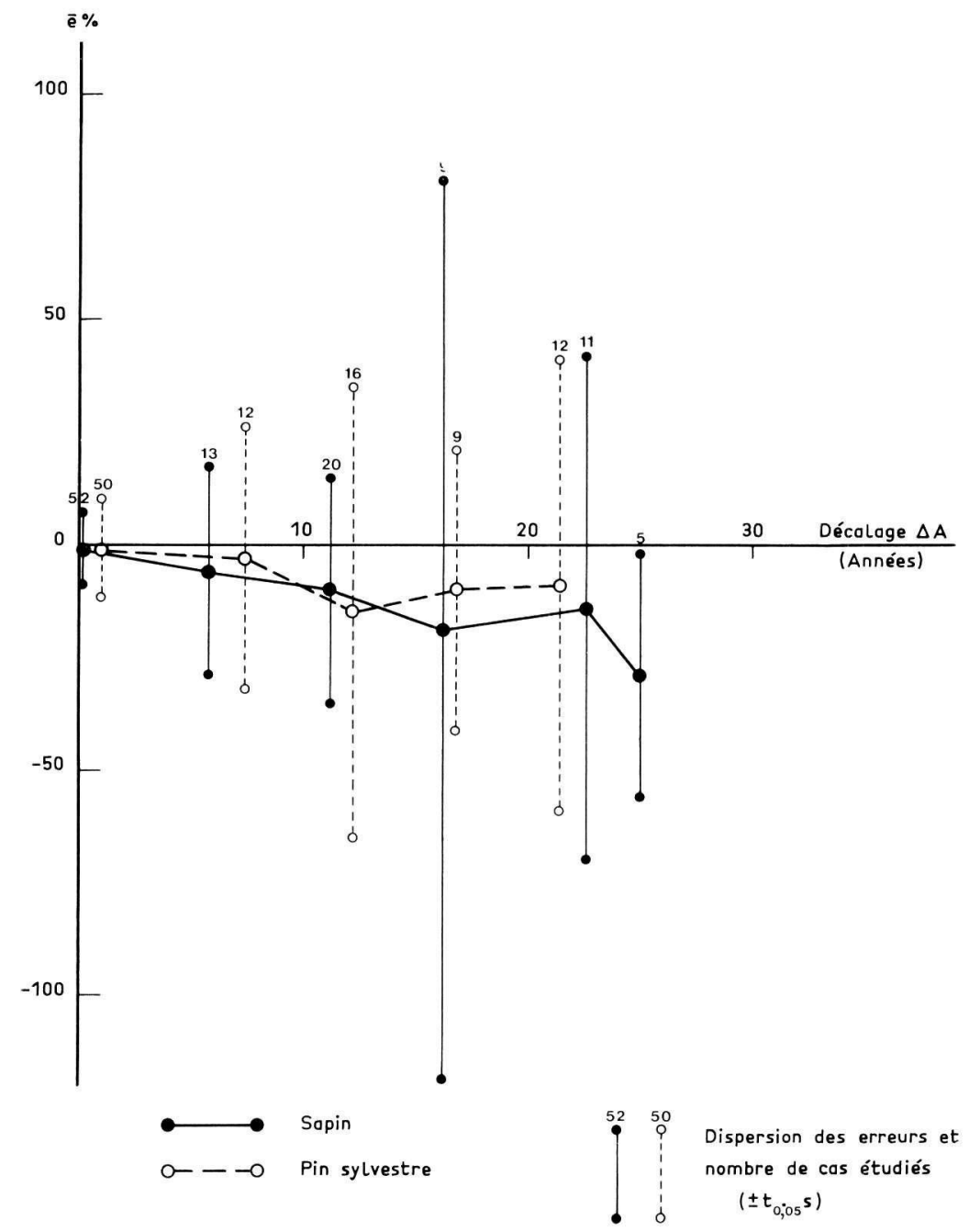

FIG. 1. - Erreur moyenne en fonction du décalage $\Delta \mathrm{A}$, entre l'établissement du tarif et l'éclaircie, pour le sapin et pour le pin sylvestre

The mean error in relation with the lag, $\Delta \mathrm{A}$, between the date of volume table construction and the thinning date, for silver fir and for scotch pine 
On a donc regroupé toutes les essences, et on s'est attaché à étudier l'influence simultanée du décalage $\Delta \mathrm{A}$ et de l'âge $\mathrm{A}$ du peuplement au moment de l'éclaircie. Les résultats des calculs figurent dans le tableau 2. La valeur des données fournie par ce tableau est évidemment très inégale et notamment la caractéristique de dispersion des erreurs observées $\pm t s$. En fait nous ne chercherons aucunement à faire une étude rigoureuse, mais seulement à situer les ordres de grandeur :

— de l'erreur moyenne $\bar{e}(\mathrm{en} \%)$;

- de la dispersion des erreurs, caractérisée par la valeur $\pm t s$, en indiquant également le décalage moyen $\Delta \mathrm{A}$ et le nombre de données utilisées pour le calcul $(n)$.

L'erreur moyenne chiffre le biais à craindre si on pratique systématiquement la méthode, par exemple lors d'un inventaire, ou pour des études de production. La dispersion (au seuil 0,05) donne une idée, de l'erreur à craindre lorsque l'opération est pratiquée une seule fois, dans un peuplement donné.

\section{2. - L'erreur moyenne (cf figure 2)}

L'erreur moyenne à craindre croît avec le décalage $\Delta \mathrm{A}$, mais l'influence de l'Age au moment de l'éclaircie est très nette. L'erreur passe par un maximum pour des âges situés entre 30 et 50 ans,

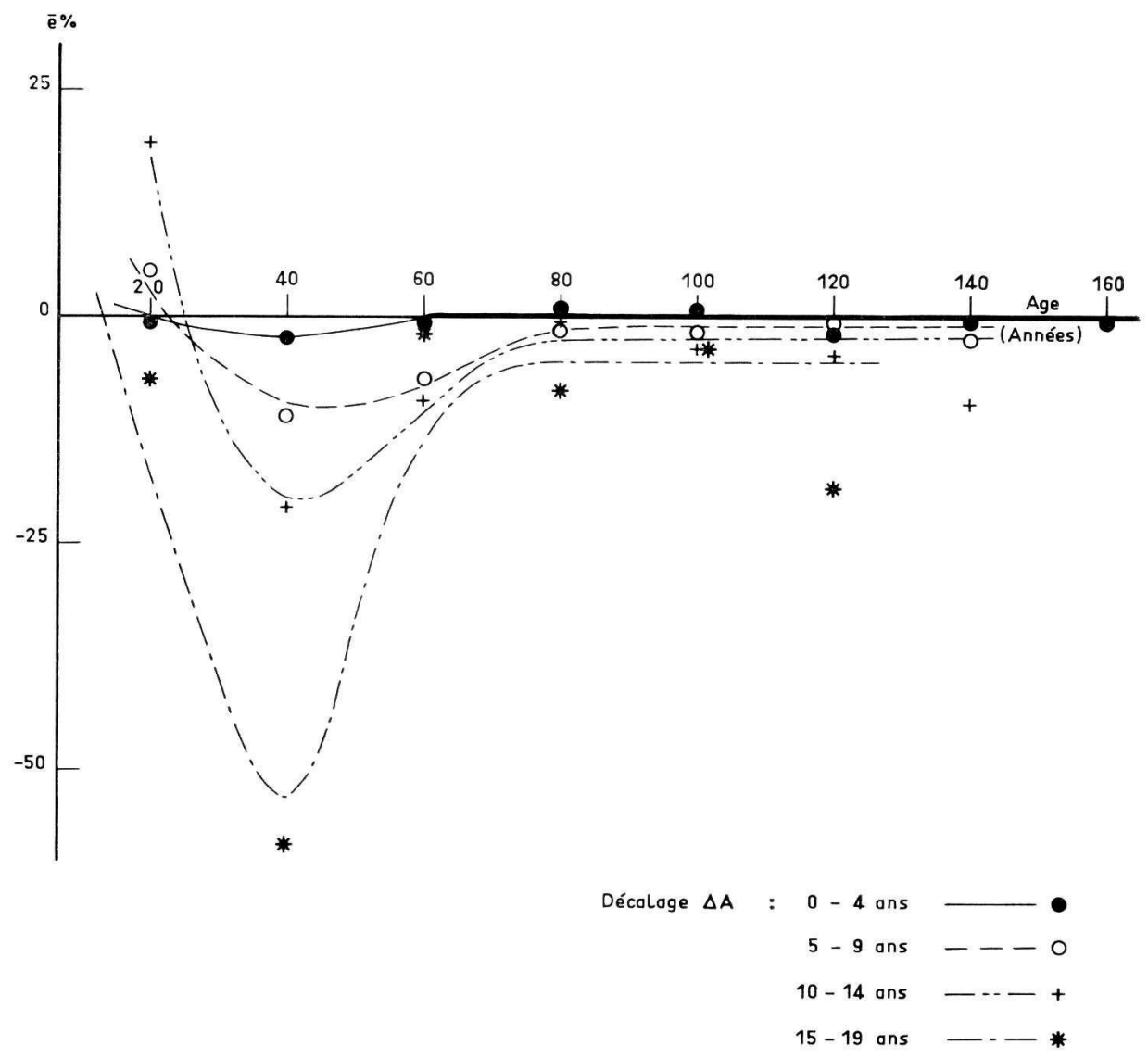

Fig. 2. - Erreur moyenne en fonction du décalage $\Delta \mathrm{A}$ et de l'âge au moment de l'éclaircie Relationship between the mean error, the lag $\Delta \mathrm{A}$ and the age at the date of the thinning 
TABLEAU 2

Résultats par décalages $\Delta \mathrm{A}$ et par âges au moment de l'éclaircie $\mathrm{A}$ (voir Texte)

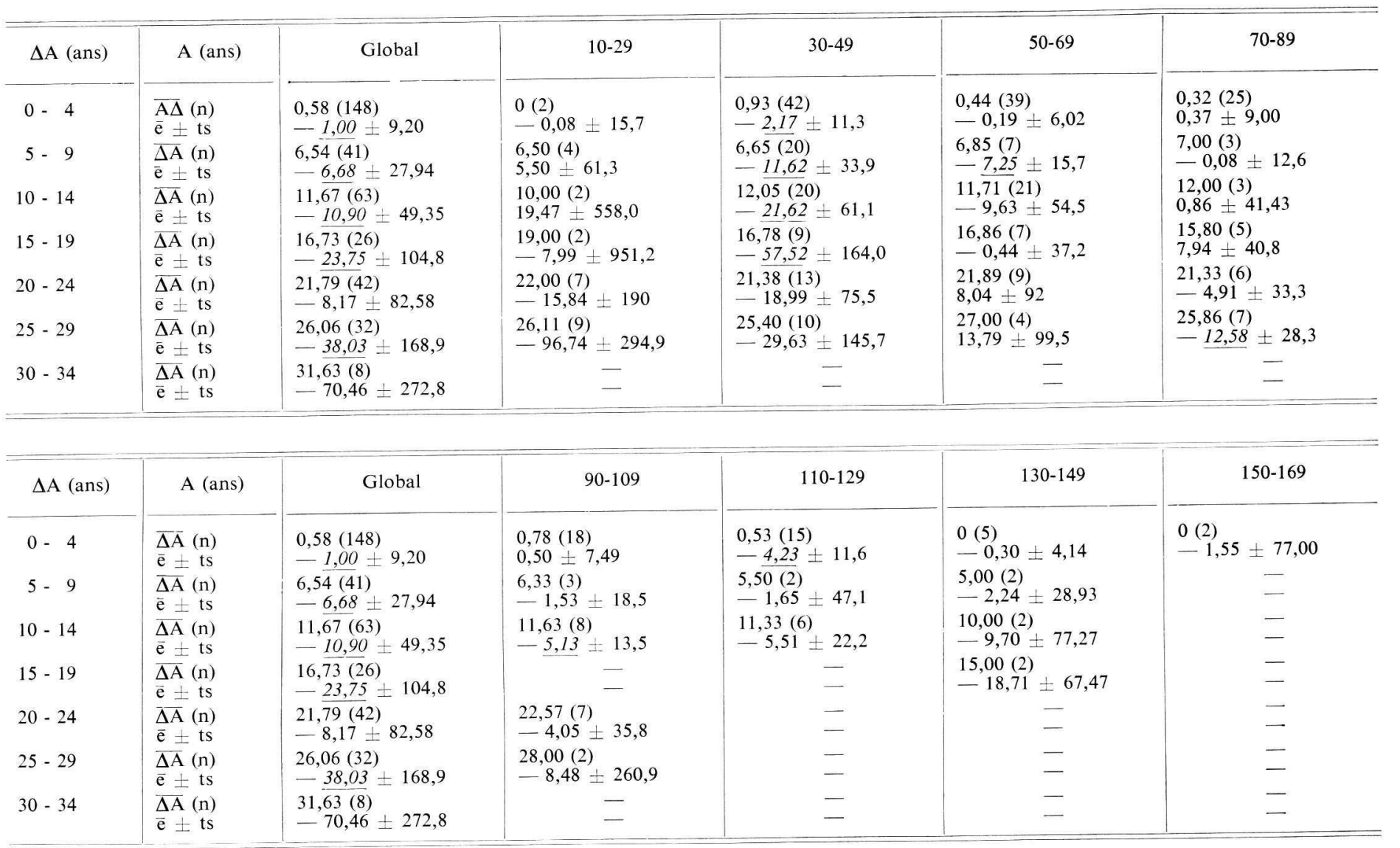

$\mathrm{N}=360$ valeurs; le $t$ utilisé correspond au seuil de 0,05 ; les ē significativement différents de zéro sont soulignés. 
c'est-à-dire finalement aux époques où les accroissements courants sont très forts notamment en hauteur, et où la forme des tiges se modifie rapidement. Dans les vieux peuplements le biais est faible, et rarement significatif, compte-tenu de la grande dispersion des résultats.

Le sens de l'erreur confirme bien que les tarifs « montent " avec l'âge.

Enfin, il semble que dans les peuplements très jeunes, l'erreur reste faible et même que son sens ait tendance à s'inverser. Nous essaierons plus loin d'interpréter ce fait.

En résumé, on peut sans doute avancer les ordres de grandeur suivants?

$$
\begin{aligned}
& \Delta \mathrm{A} \leqslant 10 \text { ans quel que soit } \mathrm{A}, \bar{e} \leqslant-10 \% \\
& \Delta \mathrm{A} \leqslant 5 \text { ans } \quad \text { « } \quad \mathrm{A}, \bar{e} \leqslant-3 \% \\
& \mathrm{~A} \geqslant 75 \text { ans } \quad \text { « } \quad \Delta \mathrm{A}, \bar{e} \leqslant-5 \%
\end{aligned}
$$
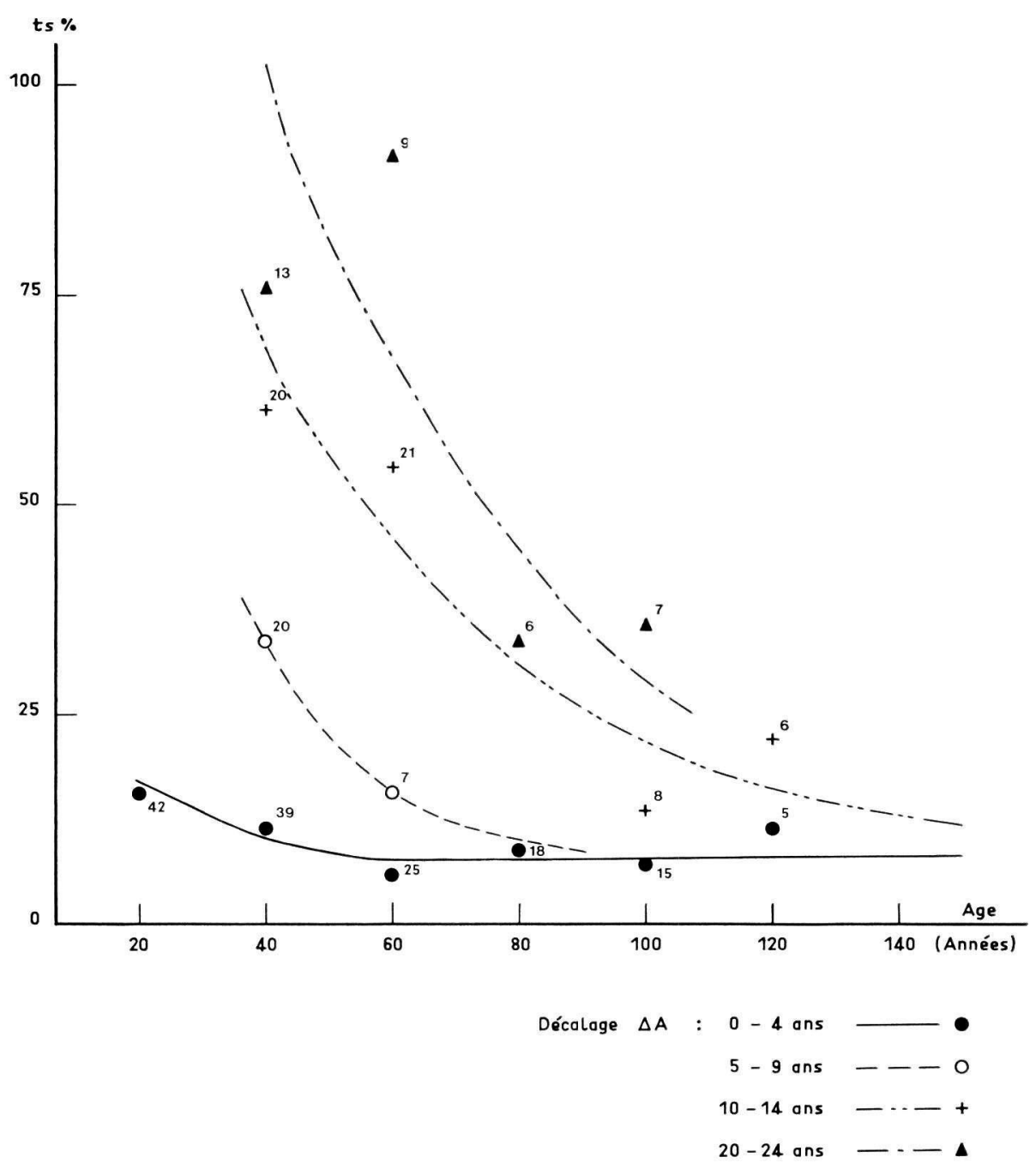

FIG. 3. - Dispersion des erreurs en fonction du décalage $\Delta \mathrm{A}$ et de l'âge au moment de l'éclaircie (le nombre de cas étudiés figure près de chaque point)

Relationship between the errors-scatter, the lag $\Delta \mathrm{A}$ and the thinning-age (the number of data is specified near each mark) 


\section{3. - La dispersion des erreurs}

La figure 3 ne comporte que des points correspondants à un nombre de couples de données supérieur à 6 et souvent supérieur à 15. C'est peu, mais nous retiendrons surtout l'allure du phénomène. La dispersion des erreurs est limitée pour de faibles décalages et pour les peuplements âgés, comme dans le cas de la moyenne des erreurs et sans que les données peu nombreuses dont on dispose permettent de mettre en évidence ce qui se passe pour les jeunes peuplements.

On peut dire approximativement que :

- pour des $\Delta \mathrm{A} \leqslant 5$ ans l'erreur à craindre sur une mesure reste de l'ordre de $\pm 10 \%$ autour de la moyenne;

- pour des $\Delta \mathrm{A} \leqslant 10$ ans cette erreur oscille entre $\pm 15 \%$ si $\mathrm{A} \geqslant 60$ ans; entre $\pm 35 \%$ si $A \geqslant 40$ ans.

Dans _tous les autres cas, l'erreur à craindre sur une mesure est considérable et difficilement prévisible.

\section{4. - Interprétation}

L'erreur commise a deux causes principales. La première est due à « la montée » des tarifs avec l'âge, la seconde au fait que les tarifs sont utilisés hors des dimensions de l'échantillon ayant servi à les construire. Cette extrapolation explique en partie la très grande variabilité de l'erreur commise, qui dépend non seulement du vieillissement du tarif, mais de la dimension des produits d'éclaircie, dimensions dont dépend l'importance de l'extrapolation pratiquée.

soit $v=a+b \mathrm{C}^{2}$, l'équation du tarif établi à l'âge Ao et servant à cuber l'éclaircie effectuée à l'âge $\mathrm{A}=\mathrm{A} o-\Delta \mathrm{A}$. A l'âge $\mathrm{A}$, le bon tarif aurait été $v^{\prime}=a^{\prime}+\mathrm{b}^{\prime} \mathrm{C}^{2}$.

Il est facile de montrer que l'erreur relative définie plus haut et commise en moyenne sur un arbre s'exprime ainsi :

$$
e=\frac{b^{\prime}-b}{b^{\prime}}+\frac{b a^{\prime}-a b^{\prime}}{b^{\prime}} \frac{1}{v^{\prime}}
$$

Si on suppose, ce qui est fréquent que les tarifs se coupent en un point de coordonnées positives $\left(\mathrm{C} o, v_{\mathrm{o}}\right)$ : on aura non seulement $b^{\prime}<b$, mais également $a^{\prime}>a$ et par conséquent :

$$
\frac{b^{\prime}-b}{b^{\prime}}<o \text { et } \frac{b a^{\prime}-a b^{\prime}}{b^{\prime}}>o
$$

On constate alors que :

- Si le volume unitaire des arbres cubés est grand, l'erreur tend vers une limite négative ne dépendant que de la pente des tarifs. Ce cas pourrait correspondre (au signe de l'erreur près) au cubage d'un peuplement sur pied avec un tarif périmé, c'est-à-dire établi plusieurs années avant le cubage.

- Si au contraire, le volume des arbres cubés est petit, l'erreur $e$ peut devenir positive et très importante.

- De plus, pour tous les arbres de volume $v>v o$ l'erreur est négative, alors qu'elle est positive pour tous ceux de volume $v<v o$ et nulle si $v=v o$. Selon le volume des arbres qui composent l'éclaircie, l'erreur de volume de cette dernière sera donc négative, positive ou nulle. Le point de croisement des tarifs étant généralement hors de leur zone de validité, ces cas correspondent bien à des extrapolations, alors qu'en se tenant dans la zone de validité l'erreur reste négative.

Pour les très jeunes peuplements cependant, la «montée » est si rapide, qu'il doit être fréquent de se trouver au voisinage du point de croisement, ce qui explique, la diminution de l'erreur moyenne constatée au paragraphe 3.2. 


\section{IV. - CONCLUSIONS PRATIQUES}

Le volume inconnu d'une éclaircie passée dont on ne retrouve que les souches ne peut être estimé valablement (biais faible, erreur à craindre inférieure à $10 \%$ ) que si elle a eu lieu il y a moins de 5 ans. $\mathrm{Si}$ la coupe date de moins de 10 ans, les erreurs sont encore acceptables (biais encore faible, erreur souvent inférieure à $25 \%$ ).

Au-delà, et sauf pour les peuplements très âgés, l'entreprise est hasardeuse. Pour les peuplemenst âgés cependant nos résultats sont intéressants dans certains cas d'expertise et aussi pour le cubage sur pied de ces peuplements à l'aide de tarifs qui bien qu'établis souvent il y a longtemps restent valablement utilisables.

Reçu pour publication en novembre 1972.

\section{SUMMARY}

\section{ERROR COMING FROM THE VOLUME TABLE IN THE ESTIMATION} OF THE VOLUME OF A THINNING BY MEASURING THE STUMPS

It is only possible to get an unbiased and fairly precise estimation (error less than $10 \%$ ) of the volume of a thinning by measuring the stumps, if the stand has been thinned for less than five years. After this period, estimations are no longer good unless the stands are old and have a slow growth.

\section{ZUSAMMENFASSUNG}

\section{TarifBedingte Fehler in der Massenschatzung von Durchforstungen VON DENEN NUR DIE StOCKDimensionen, BEKANNT SIND}

Das unbekannte Volumen einer Durchfortsung von welcher man nur die Stockumfänge kennt, kann nur dann mit hinreichender. Genauigkeit (Fehler $<10 \%$ u. geringer systematischer Fehler) bestimmt werden, wenn in jungen Beständen die Durchforstung nicht mehr als 5 Jahre Zurückliegt.

Über diese Zeitspanne hinaus kann die Schätzung der Durchforstungs masse nur in alten Beständen mit geringem Zuwachs durchgeführt werden.

\section{RÉFÉRENCES BIBLIOGRAPHIQUES}

Decourt N. 1964. - Remarques sur la relation entre les circonférences à hauteur d'homme et les circonférences à hauteur de souche dans les peuplements forestiers. R.F.F. mars 1964 - p. 216-224.

PARdÉ J., 1961. - Dendrométrie. Imprimerie Louis Jean, Gap - 350 p. 